\title{
Perturbations around the AdS Born-Infeld black holes
}

\author{
Yunqi Liu, Bin Wang \\ INPAC and Department of Physics, Shanghai Jiao Tong University, Shanghai 200240, China
}

\begin{abstract}
We study the quasinormal mode of the perturbation of the scalar field interacting with the electromagnetic field in the backgrounds of Schwarzschild AdS black hole, the Reissner-Nordstrom AdS black hole and the Born-Infeld AdS black hole. We disclose influence by the electric charge $Q$, the coupling between the scalar and electromagnetic field $q$ and the Born-Infeld parameter $b$ on the quasinormal frequencies of the scalar perturbation. We observe the possible growing mode in the perturbation when the scalar field strongly couples to the electromagnetic field. When the electromagnetic field becomes nonlinear described by the Born-Infeld electrodynamics, the nonlinearity described by the Born-Infeld parameter can hinder the growing mode to appear in the scalar perturbation. The quasinormal mode of the scalar field perturbation can help us further understand the physics in the holographic superconductor.
\end{abstract}

PACS numbers: $11.25 . \mathrm{Tq}, 04.70 . \mathrm{Bw}, 74.20 .-\mathrm{z}$ 


\section{INTRODUCTION}

Quasinormal mode (QNM) describes the perturbations in the surrounding geometry of a black hole. Its frequency is entirely fixed by the black hole parameters. The QNM is believed as a unique fingerprint of the black hole existence (for reviews on this topic, see [1 4] and references therein). In the last decade, QNM has become an intriguing subject. It can serve as a testing ground of fundamental physics. It is widely believed that the study of QNM can help us get deeper understandings of the AdS/CFT[3-7], dS/CFT [8] correspondences, loop quantum gravity [9], the phase transition of black holes [10] and the extra dimension [1] etc.

In most studies of the QNM, the wave dynamics of a single classical field propagating outside the black hole is taken into account. For a stable black hole background, the perturbation of a single classical field will die out finally due to the black hole absorption. Recently more interest has been focused on the strongly coupled theory by considering the Einstein-Maxwell field interacting with a charged scalar field. It has been shown that the bulk AdS black hole can become unstable and scalar hair can condense below a critical temperature. In the light of AdS/CFT correspondence, it was realized that the instability of the bulk black hole corresponds to a second order phase transition from normal state to superconducting state which brings the spontaneous $U(1)$ symmetry breaking [12]. Along this line, there have been a lot of investigations concerning the application of AdS/CFT correspondence to condensed matter physics by considering interactions among classical fields

[13]-[38]. It was found that the QNM can be an effective tool to disclose the second order transition between a non-superconducting state at high temperatures and a superconducting state at low temperatures.

In this work we will consider the nonlinear extension on the gauge fields in Einstein-Maxwell theory and investigate the perturbation of the scalar field in interaction with the nonlinear electromagnetic field. Recently in the bulk Schwarzschild AdS black hole and the AdS Gauss-Bonnet black hole backgrounds, the effect of the Born-Infeld electrodynamics on the holographic superconductor has been investigated in [39]. To see exactly how the higher derivative corrections to the gauge matter fields influence the perturbation around the bulk black hole and make the scalar field condense, we are going to examine the QNM of the scalar perturbation when it is coupled with the Born-Infeld electromagnetic field. In our investigation we will adopt the AdS Schwarzschild and the AdS Born-Infeld black hole solutions as backgrounds.

The structure of the paper is as follows: In Sec.II, we will introduce the action and deduce the Einstein 
equation and equations of motions describing the nonlinear electromagnetic field and the charged scalar perturbation in the five-dimensional AdS Schwarzschild and Born-Infeld black hole spacetimes. In Sec.III, we will present the numerical results and disclose the properties of the QNM of the scalar perturbation when it is coupled with the nonlinear electromagnetic field. We will summarize our results in Sec.IV.

\section{ACTION AND BASIC EQUATIONS}

The general action describing the Einstein-Born-Infeld field interacting with a charged scalar field in the five-dimensional AdS background has the form

$$
S=\frac{1}{2 \kappa^{2}} \int d^{5} x \sqrt{-g}\left(R+\frac{12}{l^{2}}\right)+\int d^{5} x \sqrt{-g}\left[\frac{1}{b}\left(1-\sqrt{1+\frac{b F^{a b} F_{a b}}{2}}\right)-|\nabla \Psi-i q A \Psi|^{2}-m^{2}|\Psi|^{2}\right]
$$

where $\kappa$ is the five-dimensional gravitational constant $\kappa^{2}=8 \pi G_{5}$, and $G_{5}$ is the five-dimensional Newton constant, $g$ is the determinant of the background black hole metric, $R$ is the Ricci curvature, $l$ describes the AdS radius, $q$ is the coupling between the electromagnetic field and the scalar field, and $m$ indicates the mass of the scalar field. Here $b$ is the Born-Infeld parameter. In the limit $b \rightarrow 0$, the Born-Infeld electrodynamics reduces to the Maxwell theory.

We assume a static black hole with the metric

$$
d s^{2}=-f(r) e^{-\chi(r)} d t^{2}+\frac{d r^{2}}{f(r)}+\frac{r^{2}}{l^{2}} d \Omega_{3}^{2}
$$

where

$$
d \Omega_{3}^{2}=d \theta^{2}+\sin ^{2} \theta\left(d \phi^{2}+\sin ^{2} \phi d \psi^{2}\right)
$$

Its Hawking temperature, which will be interpreted as the temperature of the CFT, has the form

$$
T=\left.\frac{f^{\prime}(r) e^{-\chi(r) / 2}}{4 \pi}\right|_{r=r_{h}}
$$

where $r_{h}$ is the radius of the black hole horizon.

For a static solution, we assume that the electromagnetic field and the scalar field take the forms

$$
A_{t}=\phi(r) d t, \quad \Psi=\Psi(r)
$$

Varying the action (11) with respect to the metric $g_{\mu \nu}$, we can obtain the Einstein equations

$$
\begin{aligned}
& 0=\chi^{\prime}(r)+\frac{4}{3} \kappa^{2} r\left(\frac{e^{\chi(r)} q^{2} \phi(r)^{2} \Psi(r)^{2}}{f(r)^{2}}+\Psi^{\prime}(r)^{2}\right) \\
& 0=f^{\prime}(r)-\frac{2}{r}+\frac{2 f(r)}{r}-\frac{4 r}{l^{2}}+\frac{2}{3} \kappa^{2} r\left[m^{2} \Psi(r)^{2}+\frac{e^{\chi(r)} q^{2} \phi(r)^{2} \Psi(r)^{2}}{f(r)}+\frac{1}{b}\left[\left(1-b \phi^{\prime}(r)^{2}\right)^{-1 / 2}-1\right]\right]
\end{aligned}
$$


The prime denotes the derivative with respect to $r$.

In the probe limit when $\kappa^{2} \rightarrow 0$, the solution of the Einstein equation gives the Schwarzschild AdS black hole with

$$
f(r)=1+\frac{r^{2}}{l^{2}}-\frac{r_{h}^{2}}{r^{2}}\left(1+\frac{r_{h}^{2}}{l^{2}}\right)
$$

where $r_{h}$ is the black hole horizon obtained from $f\left(r_{h}\right)=0$.

For the nonzero parameter $\kappa^{2}$, we take the backreaction of classical fields onto the spacetime into account. In studying the perturbationof the massless scalar field $\Psi(r)$ around the black hole, we can reduce Eq.(44) into

$$
\begin{aligned}
& 0=\chi^{\prime}(r) \\
& 0=f^{\prime}(r)-\frac{2}{r}+\frac{2 f(r)}{r}-\frac{4 r}{l^{2}}+\frac{2}{3 b} \kappa^{2} r\left[\left(1-b \phi^{\prime}(r)^{2}\right)^{-1 / 2}-1\right] .
\end{aligned}
$$

If we know the behavior of the electromagnetic field, we can obtain the black hole metric from the above Einstein equations.

The equation governing electromagnetic field can be obtained by varying the action (1) with respect to $\phi$, which reads

$$
\begin{aligned}
0= & {\left[\phi^{\prime \prime}(r)+\left(\frac{\chi^{\prime}(r)}{2}+\frac{3}{r}\right) \phi^{\prime}(r)\right]\left(1-b e^{\chi(r)} \phi^{\prime}(r)^{2}\right) } \\
& +\frac{b}{2} \phi^{\prime}(r) e^{\chi(r)}\left(\chi^{\prime}(r) \phi^{\prime}(r)^{2}+2 \phi^{\prime}(r) \phi^{\prime \prime}(r)\right)-\frac{2 q^{2} \phi(r) \Psi(r)^{2}}{f(r)}\left(1-b e^{\chi(r)} \phi^{\prime}(r)^{2}\right)^{3 / 2}
\end{aligned}
$$

Considering the perturbation of the massless scalar field $\Psi(r)$, Eq. (6) reduces to

$$
0=\left[\phi^{\prime \prime}(r)+\left(\frac{\chi^{\prime}(r)}{2}+\frac{3}{r}\right) \phi^{\prime}(r)\right]\left(1-b e^{\chi(r)} \phi^{\prime}(r)^{2}\right)+\frac{b}{2} \phi^{\prime}(r)^{2} e^{\chi(r)}\left(\chi^{\prime}(r) \phi^{\prime}(r)+2 \phi^{\prime \prime}(r)\right) .
$$

Doing the rescales

$$
\begin{gathered}
e^{\chi} \rightarrow a^{2} e^{\chi}, \phi \rightarrow a^{-1} \phi, t \rightarrow a t, \omega \rightarrow a^{-1} \omega . \\
r \rightarrow r / l, q \rightarrow q l, \omega \rightarrow l \omega, \phi \rightarrow \phi / l \\
q \rightarrow a q, \phi \rightarrow \phi / a, \kappa^{2} \rightarrow \kappa^{2} a^{2}, b \rightarrow a^{2} b,
\end{gathered}
$$

where the first symmetry guarantees $\chi(r)=0$, the second symmetry sets AdS radius as unity, the third one relates the backreaction to the charge of the scalar field and we can set $\kappa=1$ without loss of generality when the backreaction is not null.

Substituting $\chi(r)=0$, Eq.(17) becomes

$$
0=b \phi^{\prime}(r)^{2} \phi^{\prime \prime}(r)+\left(1-b \phi^{\prime}(r)^{2}\right)\left(\frac{3 \phi^{\prime}(r)}{r}+\phi^{\prime \prime}(r)\right) .
$$


We can solve this equation of motion of the electromagnetic field analytically, which reads [40]

$$
\phi(r)=U+\frac{Q}{r^{2}} F_{2}\left[\frac{1}{3}, \frac{1}{2}, \frac{4}{3},-\frac{4 b Q^{2}}{r^{6}}\right]
$$

where $Q$ and $U$ are integral constants, $U$ can be eliminated by the constraint at the horizon, $\phi\left(r_{h}\right)=0$,

$$
U=-\frac{1}{r_{h 2}^{2}} F_{1}\left[\frac{1}{3}, \frac{1}{2}, \frac{4}{3},-\frac{4 b Q^{2}}{r^{6}}\right] .
$$

At infinity, Eq.(10) asymptotically behaves as

$$
\phi(r) \sim U-\frac{Q}{r^{2}}
$$

In the limit $b \rightarrow 0$, Eq.(9) is reduced to

$$
0=\frac{3 \phi^{\prime}(r)}{r}+\phi^{\prime \prime}(r)
$$

which is the equation of motion of the electromagnetic field in the Maxwell theory. The solution reads

$$
\phi(r)=\frac{Q}{r_{h}^{2}}-\frac{Q}{r^{2}} .
$$

Substituting Eq.(10) into Eq.(15), the differential equation of $f(r)$ is rewritten as

$$
0=f^{\prime}(r)+\frac{2 f(r)}{r}-\frac{2}{r}-\frac{4 r}{l^{2}}-\frac{2 \kappa^{2} r}{3 b}+\frac{2 \kappa^{2}}{3 b r^{2}} \sqrt{4 b Q^{2}+r^{6}}
$$

which has an analytical solution

$$
f(r)=1+\frac{P}{r^{2}}+\frac{r^{2}}{l^{2}}+\frac{\kappa^{2} r^{2}}{6 b}-\frac{2 \kappa^{2}}{3 b r^{2}} \int \sqrt{4 b Q^{2}+r^{6}} d r .
$$

After the integration, we obtain

$$
f(r)=1+\frac{P}{r^{2}}+\frac{r^{2}}{l^{2}}+\frac{\kappa^{2} r^{2}}{6 b}-\frac{\kappa^{2}}{6 b r} \sqrt{4 b Q^{2}+r^{6}}+\frac{\kappa^{2} Q^{2}}{r^{4}} F_{2}\left[\frac{1}{3}, \frac{1}{2}, \frac{4}{3},-\frac{4 b Q^{2}}{r^{6}}\right],
$$

where $P$ is the integration constant determined by the boundary condition at the horizon $r_{h}$,

$$
P=-r_{h}^{2}\left(1+\frac{r_{h}^{2}}{l^{2}}\right)-\frac{\kappa^{2} r_{h}^{4}}{6 b}+\frac{\kappa^{2} r_{h}^{4}}{6 b} \sqrt{r_{h}^{6}+4 b Q^{2}}-\frac{\kappa^{2} Q^{2}}{r_{h}^{2}} F_{2}\left[\frac{1}{3}, \frac{1}{2}, \frac{4}{3},-\frac{4 b Q^{2}}{r^{6}}\right]
$$

Eq.(14) is exactly the solution of the five-dimensional Born-Infeld-Anti-de sitter(BIAdS) black hole described in [40]. Taking the limit $b \rightarrow 0$, Eq.(12) changes into

$$
0=f^{\prime}(r)+\frac{2 f(r)}{r}-\frac{2}{r}-\frac{4 r}{l^{2}}+\frac{4 \kappa^{2} Q^{2}}{3 r^{5}}
$$

which gives the Reissner-Nordstrom AdS black hole with the metric coefficient

$$
f(r)=1+\frac{r^{2}}{l^{2}}+\frac{2 \kappa^{2} Q^{2}}{3 r^{4}}\left(1-\frac{r^{2}}{r_{h}^{2}}\right)-\frac{r_{h}^{2}}{r^{2}}\left(1+\frac{r_{h}^{2}}{l^{2}}\right) .
$$


Thus in contrast to the bakcground in the probe limit Eq.(15), we can see that the backreaction of the electromagnetic field on the Einstein gravity makes the black hole charged.

Now we consider the scalar field perturbing around the AdS black hole background (2), we have the wave equation of the charged scalar field directly from the action

$$
0=\psi^{\prime \prime}(r)+\psi^{\prime}(r)\left[\frac{3}{r}+\frac{f^{\prime}(r)}{f(r)}-\frac{\chi^{\prime}(r)}{2}\right]+\psi(r) \frac{(\omega+q \phi(r))^{2} e^{\chi(r)}}{f(r)^{2}}
$$

where we have separated the radial part of the scalar field into $\Psi(r)=\psi(r) e^{-i \omega t}$. Here $\omega$ indicates the frequency of the perturbation.

Near the black hole horizon $r \sim r_{h}$, we can impose the incoming wave boundary condition

$$
\psi(r) \sim\left(r-r_{h}\right)^{-i \frac{\omega}{4 \pi T}}
$$

Introducing a new variable $\varphi$, we separate $\psi(r)=\Re(r) \varphi(r)$ where $\Re(r)=\exp \left[-i \int_{r_{h}}^{r} \frac{\omega+q \phi(r)}{f(r)}\right]$, which asymptotically approaches Eq.(19) at the horizon. Then at the horizon $\left.\varphi\right|_{r=r_{h}}=$ const. and Eq.(18) can be expressed as

$$
0=\varphi^{\prime \prime}(r)+B_{1}(r) \varphi^{\prime}(r)+B_{2}(r) \varphi(r)
$$

with

$$
\begin{aligned}
& B_{1}(r)=\frac{f^{\prime}(r)}{f(r)}-\frac{2 i[q \phi(r)+\omega]}{f(r)}+\frac{3}{r} \\
& B_{2}(r)=-\frac{i\left[3(q \phi(r)+\omega)+r \phi^{\prime}(r)\right]}{r f(r)}
\end{aligned}
$$

At the spatial infinity $r \sim \infty, \varphi(r)$ behaves as

$$
\varphi(r) \sim \frac{\varphi_{-}}{r^{\lambda_{-}}}+\frac{\varphi_{+}}{r^{\lambda_{+}}}
$$

where $\lambda_{ \pm}=d / 2 \pm\left(d / 2+m^{2} l^{2}\right)^{1 / 2}=2 \pm 2$ for taking $m=0, d=4$. We choose $\varphi_{-}=0$ in our following discussion, so that we can only relate the scalar operator in the field theory dual to the branch $\varphi_{+}$to describe the condensation.

We can set the boundary conditions at the horizon

$$
\begin{aligned}
\left.\varphi\right|_{r=r_{h}} & =1 \\
\left.\frac{\varphi^{\prime}}{\varphi}\right|_{r=r_{h}} & =-\left.\frac{B_{2}(r)}{B_{1}(r)}\right|_{r=r_{h}}
\end{aligned}
$$

to solve Eq.(201). 
Substituting $f(r)$ and $\phi(r)$ obtained from solving the Einstein equation and the electromagnetic equation into Eq.(20), we can get the differential equation governing the perturbation of scalar field outside the AdS black hole. Using the boundary conditions (23) and imposing $\varphi_{-}=0$ at infinity, we can calculate the frequency $\omega$ of the scalar perturbation. We will carry out the numerical computation by using the shooting method and disclose the properties of the QNM of the massless charged scalar perturbation when it is coupled to the nonlinear electromagnetic field.

\section{NUMERICAL RESULTS}

In this section we present our numerical results of the scalar perturbation. We will first focus on the perturbation in the background of the Schwarzschild AdS black hole. When there is no coupling between the scalar field and the electromagnetic field, namely $q=0$, we can reproduce the QNM of the single scalar field

perturbation obtained in [41]. This shows that our shooting method is effective in the numerical calculation.

Now we turn on the coupling parameter $q$, the quasinormal frequencies of the scalar perturbation when it is coupled with the electric field around the Schwarzschild AdS black hole are listed in table I. In obtaining the numerical results, we have fixed the black hole size $r_{h}=0.1$ and the charge of the electromagnetic field $Q=0.1$. When $b=0$, our result goes back to [42]. For other fixed nonzero Born-Infeld parameter $b$, we see the same qualitative feature that with the increase of the coupling parameter $q$, both the real part and the absolute value of the imaginary part of the quasinormal frequencies become smaller. This shows that when the coupling between the scalar field and electromagnetic field is stronger, the scalar perturbation on background spacetime decays slower. When $q$ is large enough, the imaginary part of the QNM can become positive which indicates that the background spacetime becomes unstable. To see the nonlinear effect of the electromagnetic field, we can fix the coupling parameter $q$ and find that with the increase of the parameter $b$, both the real part and the absolute value of the imaginary part of the quasinormal frequencies become bigger. This shows that when there is coupling between the scalar field and the electromagnetic field, the nonlinearity of the electromagnetic field can influence the scalar field perturbation and make it decay faster. Furthermore we observe that when the Born-Infeld parameter is bigger, it needs stronger coupling $q$ to destroy the bulk spacetime to make it unstable.

From now on we concentrate on the Born-Infeld AdS black hole spacetime. When the Born-Infed parameter 
TABLE I: The dependence of the QNM on $q$ and $b$ for Schwarzschild AdS BH with $r_{h}=0.1, Q=0.1$.

\begin{tabular}{l|l|l}
\hline \hline $\mathrm{q}$ & $\mathrm{b}$ & $\omega$ \\
\hline 0.1 & 0 & $2.968076-0.011512 i$ \\
& 0.001 & $3.463266-0.013715 i$ \\
& 0.002 & $3.539744-0.014161 i$ \\
& 0.003 & $3.580766-0.014419 i$ \\
& 0.004 & $3.608080-0.014598 i$ \\
\hline 0.3 & 0 & $1.029025-0.002924 i$ \\
& 0.001 & $2.514249-0.007662 i$ \\
& 0.002 & $2.743949-0.008586 i$ \\
& 0.003 & $2.867207-0.009126 i$ \\
& 0.004 & $2.949297-0.009507 i$ \\
\hline 0.5 & 0 & $-0.915499+0.001992 i$ \\
& 0.001 & $1.560090-0.003788 i$ \\
& 0.002 & $1.943326-0.004890 i$ \\
& 0.003 & $2.149033-0.005538 i$ \\
& 0.004 & $2.286064-0.005998 i$ \\
\hline 0.7 & 0.001 & $0.601607-0.001210 i$ \\
& 0.002 & $1.138613-0.002375 i$ \\
& 0.003 & $1.426927-0.003055 i$ \\
& 0.004 & $1.619019-0.003538 i$ \\
\hline 0.9 & 0.001 & $-0.360504+0.000625 i$ \\
& 0.002 & $0.330434-0.000590 i$ \\
& 0.003 & $0.701466-0.001283 i$ \\
& 0.004 & $0.948705-0.001770 i$ \\
\hline \hline 1.1 & 0.002 & $-0.480700+0.000759 i$ \\
& 0.003 & $-0.026874+0.000043 i$ \\
& 0.004 & $0.275571-0.000450 i$ \\
\hline & 0.003 & $-0.757706+0.001010 i$ \\
& 0.004 & $-0.400013+0.000586 i$ \\
\hline
\end{tabular}

tends to zero, the spacetime boils down to the Reissner-Nordstrom AdS black hole. For the Reissner-Nordstrom AdS black hole, we know that there is an upper bound of the electric charge contained inside the black hole, $Q_{e x}=\sqrt{\frac{3+6 r_{h}^{2}}{2}} \frac{r_{h}^{2}}{\kappa}$. For the Born-Infeld AdS black hole, this extreme charge $Q_{e x}$ can be calculated numerically.

When we choose the coupling parameter $q$ between the scalar field and the electric field to be zero and the Born-Infeld parameter $b=0$, we can obtain the QNM for the single scalar perturbation in the ReissnerNordstrom AdS background as listed in table II, which is consistent with the computation in [6]. When the Reissner-Nordstrom AdS black hole becomes more charged, the real part of the quasinormal frequency decreases while the imaginary part becomes more negative. Keeping $q=0$ and turning on the Born-Infeld parameter $b$, we can have the QNM of the single scalar field in the Born-Infeld AdS black hole as shown in table III. The nonlinear property of the electric field will not change the dependence of the quasinormal frequency on the black hole charge. 
TABLE II: $\kappa=1, q=0, b=0$. The dependence of the QNM on Q and $r_{h}$ around the Reissner-Nordstrom AdS black hole.

\begin{tabular}{c|c|c|c}
\hline \hline$r_{h}$ & $Q$ & $Q / Q_{e x}$ & $\omega$ \\
\hline 0.1 & 0.00123 & 0.1 & $3.934866-0.018082 i$ \\
& 0.00247 & 0.2 & $3.932722-0.018429 i$ \\
& 0.00495 & 0.4 & $3.923931-0.020024 i$ \\
& 0.00742 & 0.6 & $3.908544-0.023703 i$ \\
& 0.00990 & 0.8 & $3.886169-0.032180 i$ \\
& 0.01113 & 0.9 & $3.873996-0.039081 i$ \\
& 0.01175 & 0.9 & $3.867630-0.043017 i$ \\
\hline 0.2 & 0.00509 & 0.1 & $3.788329-0.168287 i$ \\
& 0.01018 & 0.2 & $3.781288-0.173062 i$ \\
& 0.02036 & 0.4 & $3.753501-0.194535 i$ \\
& 0.03055 & 0.6 & $3.711659-0.239103 i$ \\
& 0.04073 & 0.8 & $3.673163-0.310469 i$ \\
& 0.04582 & 0.9 & $3.658485-0.350673 i$ \\
& 0.04837 & 0.95 & $3.651815-0.371542 i$ \\
\hline \hline
\end{tabular}

TABLE III: The dependence of the QNM of single scalar field on the Born-Infeld AdS black hole background. Here we have chosen $b=1$.

\begin{tabular}{c|c|c|c}
\hline \hline$r_{h}$ & $Q$ & $Q / Q_{e x}$ & $\omega$ \\
\hline 0.01 & 0.00150 & 0.1 & $3.999782-0.0000123 i$ \\
& 0.00300 & 0.2 & $3.995464-0.0000125 i$ \\
& 0.00600 & 0.4 & $3.989700-0.0000129 i$ \\
& 0.00900 & 0.6 & $3.983007-0.0000135 i$ \\
& 0.01200 & 0.8 & $3.975621-0.0000146 i$ \\
& 0.01350 & 0.9 & $3.971702-0.0000158 i$ \\
& 0.01425 & 0.95 & $3.969690-0.0000175 i$ \\
\hline 0.1 & 0.01535 & 0.1 & $3.909637-0.020425 i$ \\
& 0.03070 & 0.2 & $3.868855-0.024228 i$ \\
& 0.06140 & 0.4 & $3.762048-0.037783 i$ \\
& 0.09210 & 0.6 & $3.621570-0.071016 i$ \\
& 0.12280 & 0.8 & $3.460195-0.155326 i$ \\
& 0.13815 & 0.9 & $3.388106-0.217904 i$ \\
& 0.14582 & 0.95 & $3.355319-0.252168 i$ \\
\hline \hline
\end{tabular}

Now we turn on the coupling between the scalar field and the electromagnetic field. In the ReissnerNordstrom AdS background, when the Born-Infeld parameter is zero, the scalar perturbation coupled with the electromagnetic field was studied in [42]. Here we present the property of the QNM of the scalar field with the change of its coupling strength with the electromagnetic field and the electric charge of the ReissnerNordstrom black hole. Our numerical results are shown in table IV. We find that for the nonzero $q$, the absolute value of the imaginary part of the quasinormal frequency does not keep on increasing with the increase of the black hole charge as we observed when $q=0$. It first decreases and then increases when the black hole becomes more charged. When the strength of the coupling $q$ is big enough, the imaginary frequency will keep on increasing with the increase of the black hole electric charge until it jumps to be positive, showing that the background spacetime becomes unstable. The real part keeps the same property as the case when $q=0$, 
it continues to decrease with the increase of the black hole charge, except for the big enough $q$ it becomes unphysical when the spacetime is destroyed. This property can be seen clearly in Fig.1.

TABLE IV: The frequencies of the charged scalar field when we choose the coupling with the Maxwell field $q=0.5,2$ and 4 respectively around the RN-AdS black hole with $r_{h}=0.1$.

\begin{tabular}{l|l|l|c}
\hline \hline$q$ & $Q$ & $Q / Q_{e x}$ & $\omega$ \\
\hline 0.5 & 0 & 0 & $3.935576-0.017971 i$ \\
& 0.00124 & 0.1 & $3.875069-0.017627 i$ \\
& 0.00247 & 0.2 & $3.813143-0.017503 i$ \\
& 0.00371 & 0.3 & $3.749777-0.017612 i$ \\
& 0.00495 & 0.4 & $3.684917-0.017997 i$ \\
& 0.00618 & 0.5 & $3.618480-0.018750 i$ \\
& 0.00742 & 0.6 & $3.550352-0.020045 i$ \\
& 0.00866 & 0.7 & $3.480442-0.022210 i$ \\
& 0.00990 & 0.8 & $3.408885-0.025790 i$ \\
& 0.01113 & 0.9 & $3.336418-0.031127 i$ \\
& 0.01175 & 0.95 & $3.300021-0.034267 i$ \\
\hline \multirow{2}{*}{4} & 0 & 0 & $3.935576-0.017971 i$ \\
& 0.00124 & 0.1 & $3.695650-0.016306 i$ \\
& 0.00247 & 0.2 & $3.454297-0.014902 i$ \\
& 0.00371 & 0.3 & $3.211561-0.013708 i$ \\
& 0.00495 & 0.4 & $2.967456-0.012691 i$ \\
& 0.00618 & 0.5 & $2.721970-0.011838 i$ \\
& 0.00742 & 0.6 & $2.475050-0.011157 i$ \\
& 0.00866 & 0.7 & $2.226596-0.010705 i$ \\
& 0.00990 & 0.8 & $1.976435-0.010659 i$ \\
& 0.01113 & 0.9 & $1.724470-0.011570 i$ \\
& 0.01175 & 0.95 & $1.598092-0.012641 i$ \\
\hline \hline & 0 & 0 & $3.935576-0.017971 i$ \\
& 0.00124 & 0.1 & $3.456359-0.014642 i$ \\
& 0.00247 & 0.2 & $2.975567-0.011821 i$ \\
& 0.00371 & 0.3 & $2.493319-0.009374 i$ \\
& 0.00495 & 0.4 & $2.009714-0.007207 i$ \\
& 0.00618 & 0.5 & $1.524838-0.005249 i$ \\
& 0.00742 & 0.6 & $1.038765-0.003447 i$ \\
& 0.00866 & 0.7 & $0.551568-0.001766 i$ \\
& 0.00990 & 0.8 & $0.063334-0.000194 i$ \\
& 0.01113 & 0.9 & $-0.425792+0.001168 i$ \\
& 0.01175 & 0.95 & $-0.670674+0.001581 i$ \\
\hline \multirow{2}{*}{4} & &
\end{tabular}

When we consider the electromagnetic field to be nonlinear with nonzero $b$, the dependence of the quasinormal frequencies of the scalar field on the coupling strength with the electromagnetic field and the electric charge $Q$ does not change. The behaviors are shown in Fig.2 for the real part and the imaginary part of the quasinormal frequencies. Comparing with the zero Born-Infeld parameter case, nonzero $b$ makes the turning point of the imaginary part of the quasinormal frequency appear for smaller $Q / Q_{e x}$ when $q$ is fixed and the positive imaginary part of the frequency can appear for bigger coupling strength $q$. Fixing $q=4$, the influence of the Born-Infeld parameter is shown in Fig.3. Similar to what we have observed in the Schwarzschild 
AdS background, when the Born-Infeld parameter is smaller, the coupling between the scalar field and the electromagnetic field can make the background become unstable more easily.
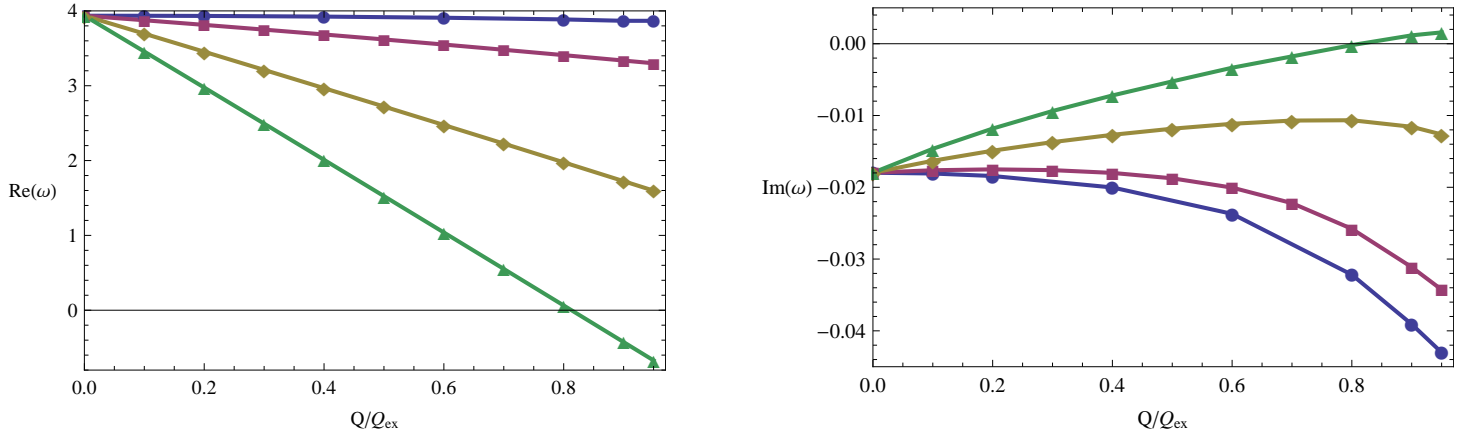

FIG. 1: The quasinormal frequencies change with the black hole charge in the Reissner-Nordstrom AdS black hole background with the black hole size $r_{h}=0.1$. For the real part of the frequency, lines from the top to the bottom correspond to $q=0,0.5,2,4$, respectively. For the imaginary part of the frequency, lines from the bottom to the top correspond to $q=0,0.5,2,4$, respectively.
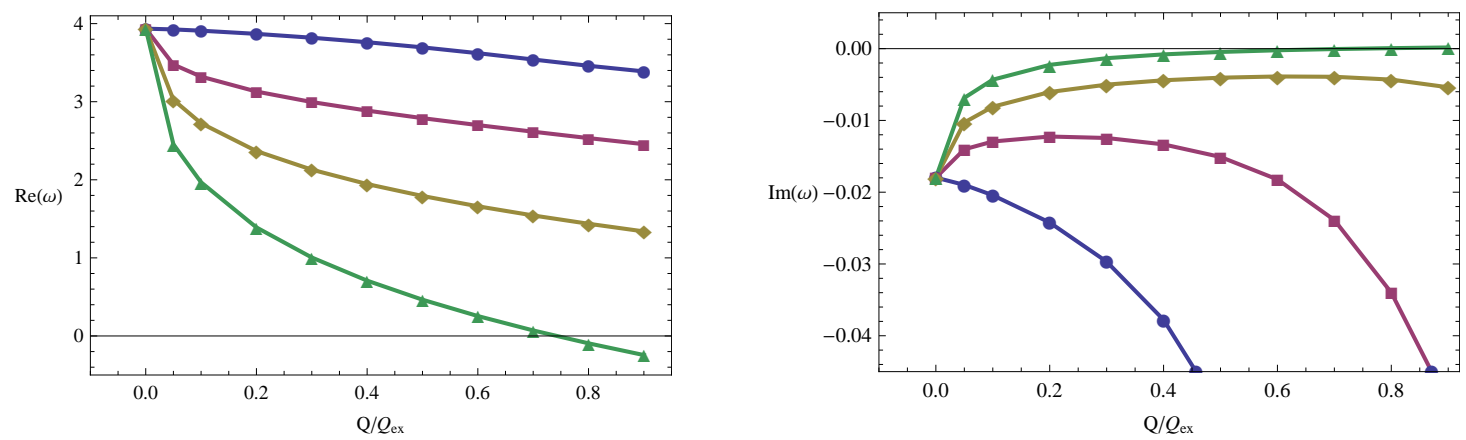

FIG. 2: The quasinormal frequencies change with the black hole charge in the Born-Infeld AdS black hole background with the Born-Infeld parameter $b=1$. The black hole size is fixed as $r_{h}=0.1$. For the real part of the frequency, lines from the top to the bottom correspond to $q=0,2,4,6.5$, respectively. For the imaginary part of the frequency, lines from the bottom to the top correspond to $q=0,2,4,6.5$, respectively.
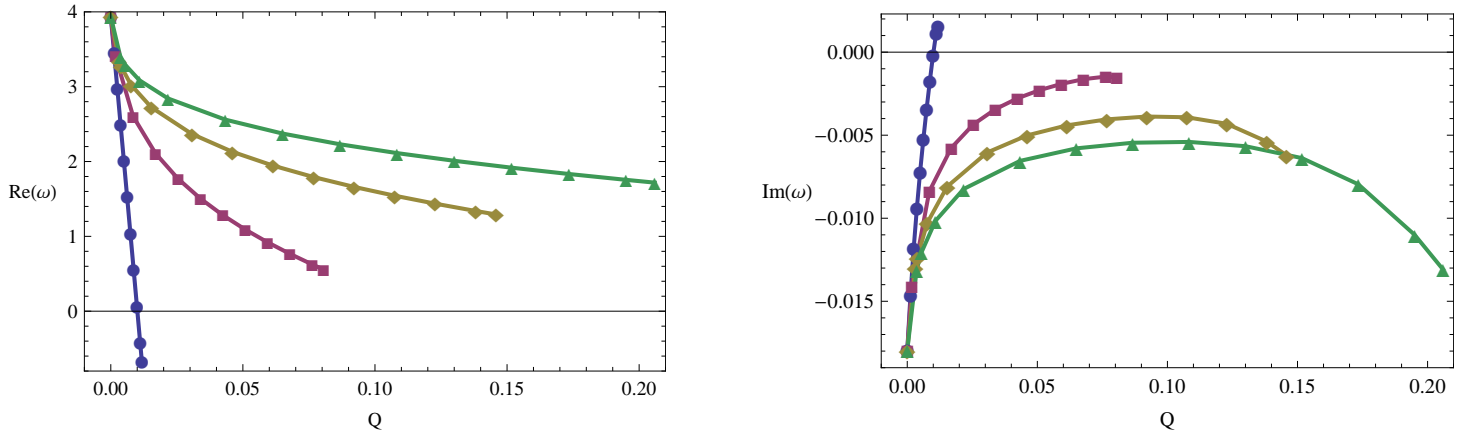

FIG. 3: The quasinormal frequencies change with the black hole charge in the AdS black hole background with the $q=4$ fixed and varying Born-Infeld parameter. The black hole size is fixed as $r_{h}=0.1$. For the real part of the frequency, lines from the top to the bottom correspond to $b=2,1,0.3,0$, respectively. For the imaginary part of the frequency, lines from the bottom to the top correspond to $b=2,1,0.3,0$, respectively.

From the above discussion, we learnt that the QNM of charged scalar field perturbation in the AdS back- 
ground is influenced by the electric charge $Q$, the coupling $q$ between the scalar field and the electromagnetic field and the Born-Infeld parameter $b$. To see more clearly the combined influence, we list our numerical results of the quasinormal frequencies in table $\mathrm{V}$ and table VI and compare with earlier result presented in table I. For the chosen value of $q$, we see that the real part of the frequency keeps increasing when the Born-Infeld parameter becomes bigger. The imaginary part of the frequency does not change monotonously. As shown in table $\mathrm{V}$, the absolute value of the imaginary frequency decreases first and then increases when the Born-Infeld parameter becomes bigger for small coupling case. When the coupling $q$ becomes stronger, the turning point of the imaginary frequency appears for smaller $b$. When $q$ is big enough, the imaginary frequency becomes monotonous and appears more negative when the Born-Infeld parameter is bigger as shown in table VI. This is consistent with that in table I. From the third equation in Eq.(8), we can see when $q$ is bigger, the backreaction becomes weaker. Thus as $q$ increases to be big enough the behavior should be similar to that in the probe limit. The property described above is shown in fig.4.

TABLE V: The dependence of the QNM on $q$ and $b$, where we have chosen $\kappa=1, r_{h}=0.1$, and $Q=0.01$.

\begin{tabular}{|c|c|c|c|c|c|}
\hline$q$ & b & $\omega$ & $q$ & b & $\omega$ \\
\hline 0.5 & $\begin{array}{l}0 \\
10^{-4} \\
10^{-2} \\
0.1 \\
0.5 \\
1 \\
10 \\
15 \\
20 \\
25\end{array}$ & $\begin{array}{l}3.402781-0.026176 i \\
3.405373-0.026070 i \\
3.519682-0.021947 i \\
3.673134-0.018869 i \\
3.764919-0.017945 i \\
3.796554-0.017752 i \\
3.870019-0.017575 i \\
3.878750-0.017586 i \\
3.884328-0.017597 i \\
3.888326-0.017608 i\end{array}$ & 1 & $\begin{array}{l}0 \\
10^{-4} \\
10^{-2} \\
0.1 \\
0.2 \\
0.3 \\
0.5 \\
1 \\
5 \\
10 \\
15\end{array}$ & $\begin{array}{l}2.920261-0.020304 i \\
2.925335-0.020222 i \\
3.146037-0.017604 i \\
3.437811-0.016273 i \\
3.518353-0.016169 i \\
3.561588-0.016153 i \\
3.611629-0.016171 i \\
3.671523-0.016248 i \\
3.777466-0.016567 i \\
3.810748-0.016733 i \\
3.827325-0.016831 i\end{array}$ \\
\hline 1.5 & $\begin{array}{l}0 \\
10^{-4} \\
10^{-2} \\
0.1 \\
0.2 \\
0.5 \\
1 \\
5 \\
10 \\
15\end{array}$ & $\begin{array}{l}2.437763-0.015128 i \\
2.445310-0.015078 i \\
2.772025-0.013916 i \\
3.202059-0.013993 i \\
3.320658-0.014206 i \\
3.457998-0.014562 i \\
3.546196-0.014866 i \\
3.702263-0.015613 i \\
3.751320-0.015929 i \\
3.775763-0.016108 i\end{array}$ & 2 & $\begin{array}{l}0 \\
10^{-4} \\
10^{-3} \\
10^{-2} \\
0.1 \\
0.3 \\
0.5 \\
1 \\
10 \\
20\end{array}$ & $\begin{array}{l}1.955207-0.010687 i \\
1.965213-0.010673 i \\
2.042778-0.010604 i \\
2.397601-0.010808 i \\
2.965891-0.011989 i \\
3.206688-0.012739 i \\
3.304039-0.013100 i \\
3.420584-0.013594 i \\
3.691739-0.015163 i \\
3.744733-0.015586 i\end{array}$ \\
\hline
\end{tabular}


TABLE VI: The dependence of the QNM on $q$ and $b$, where we have chosen $\kappa=1, r_{h}=0.1$ and $Q=0.01$.

\begin{tabular}{l|l|l|l}
\hline \hline $\mathrm{b}$ & $\omega(\mathrm{q}=3)$ & $\omega(\mathrm{q}=4)$ & $\omega(\mathrm{q}=5)$ \\
\hline 0 & $0.989457-0.004056 i$ & $0.022024-0.000067 i$ & $-0.947650+0.002231 i$ \\
$10^{-4}$ & $1.004346-0.004101 i$ & $0.041779-0.000127 i$ & $-0.923010+0.002183 i$ \\
$10^{-2}$ & $1.647412-0.006033 i$ & $0.895384-0.002710 i$ & $0.141586-0.000363 i$ \\
0.1 & $2.492360-0.008681 i$ & $2.017336-0.006116 i$ & $1.540944-0.004112 i$ \\
0.5 & $2.975184-0.010568 i$ & $2.685157-0.008475$ & $2.374046-0.006739 i$ \\
1 & $3.168540-0.011349 i$ & $2.915465-0.009446 i$ & $2.661429-0.007831 i$ \\
5 & $3.475528-0.013057 i$ & $3.323476-0.011580 i$ & $3.170748-0.010263 i$ \\
10 & $3.572124-0.013735 i$ & $3.451928-0.012436 i$ & $3.331173-0.011255 i$ \\
20 & $3.651069-0.014369 i$ & $3.556941-0.013243 i$ & $3.462364-0.012202 i$ \\
40 & $3.714917-0.014953 i$ & $3.641898-0.013993 i$ & $3.568529-0.013091 i$ \\
80 & $3.766023-0.015482 i$ & $3.709919-0.014677 i$ & $3.653550-0.013911 i$ \\
100 & $3.780121-0.015640 i$ & $3.728684-0.014882 i$ & $3.677008-0.014158 i$ \\
\hline \hline
\end{tabular}
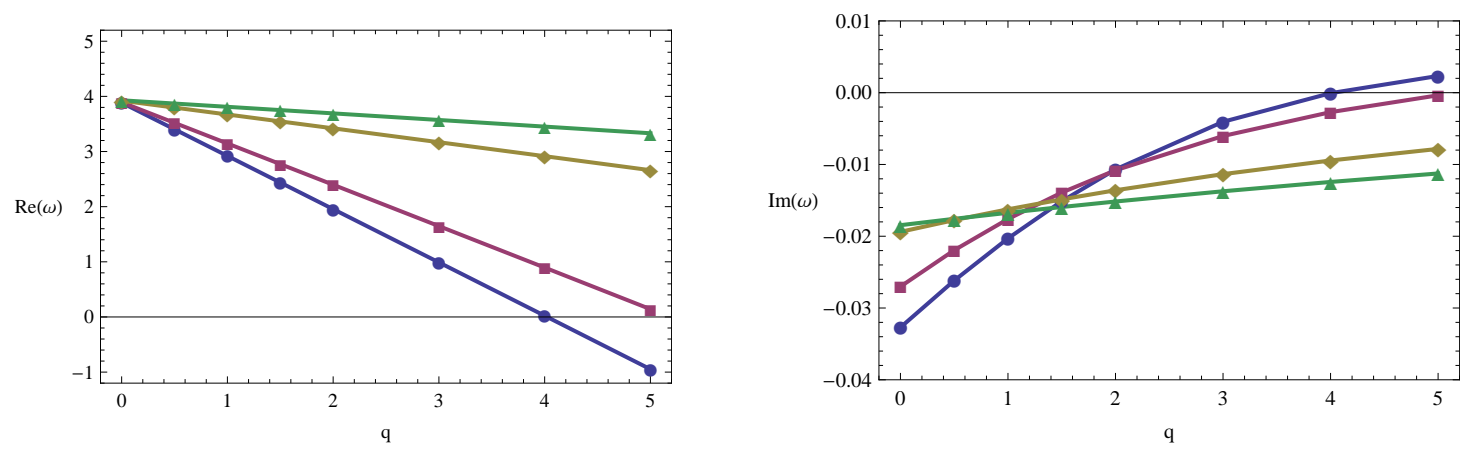

FIG. 4: The quasinormal frequencies change with the coupling parameter $q$ in the AdS black hole background for different Born-Infeld parameter $b$. The black hole size is fixed as $r_{h}=0.1$ and the electric charge is chosen as $Q=0.01$. For the real part of the frequency, lines from the top to the bottom correspond to $b=10,1,0.01,0$, respectively. For the imaginary part of the frequency, lines from the bottom to the top correspond to $b=10,1,0.01,0$, respectively.

\section{SUMMARY}

In this work we have studied the QNM of the perturbation of the scalar field interacting with the electromagnetic field in the backgrounds of neutral AdS black hole, the Reissner-Nordstrom AdS black hole and the Born-Infeld AdS black hole. We have disclosed rich physics brought by the electric charge $Q$, the coupling between the scalar and electromagnetic field $q$ and the Born-Infeld parameter $b$ on the quasinormal frequencies of the scalar perturbation. Different from the single classical field perturbation, which always has the decay mode in the black hole background, we observed the possible growing mode when the perturbation of the scalar field strongly couples to the electromagnetic field. This indicates that the interaction among classical fields can destroy the background spacetime. In the language of the AdS/CFT, this is due to the condensate of the scalar field on the black hole background and there is a second order transition between a non-superconducting state at high temperatures and a superconducting state at low temperatures. Our 
results disclose the signature of how this phase transition happens from the phenomenon of the perturbation.

When the electromagnetic field becomes nonlinear described by the Born-Infeld electrodynamics, the nonlin-

earity described by the Born-Infeld parameter can hinder the instability to happen in the scalar perturbation

around the background spacetime. The QNM behavior of the scalar field disclosed here can help us further understand the physics in the holographic superconductor.

Acknowledgment: We thank Qiyuan Pan and Ma Lei for useful discussions. This work was supported in part by NNSF of China.

[1] H. P. Nollert, Class. Quant. Grav. 16, R159 (1999).

[2] K. D. Kokkotas and B. G. Schmidt, Living Rev. Rel. 2, 2 (1999).

[3] B. Wang, Braz. J. Phys. 35,1029 (2005).

[4] R. A. Konoplya, A. Zhidenko, Rev.Mod.Phys.83, 793-836 (2011).

[5] J. S. F. Chan and R. B. Mann, Phys. Rev. D 59, 064025 (1999); G. T. Horowitz and V. E. Hubeny, Phys. Rev. D 62, $024027(2000)$.

[6] B. Wang, C. Y. Lin, and E. Abdalla, Phys. Lett. B 481, 79 (2000).

[7] B. Wang, C. Molina, and E. Abdalla, Phys. Rev. D 63, 084001 (2001); J. M. Zhu, B. Wang, and E. Abdalla, Phys. Rev. D 63, 124004 (2001); V. Cardoso and J. P. S. Lemos, Phys. Rev. D 63, 124015 (2001); V. Cardoso and J. P. S. Lemos, Phys. Rev. D 64, 084017(2001); E. Berti and K. D. Kokkotas, Phys. Rev. D 67, 064020 (2003); V. Cardoso and J. P. S. Lemos, Class. Quantum Grav. 18, 5257 (2001);E. Winstanley, Phys. Rev. D 64, 104010 (2001); J. Crisstomo, S. Lepe and J. Saavedra, Class. Quant. Grav. 21, 2801-2810 (2004); S. Lepe, F. Mendez, J. Saavedra, L. Vergara, Class.Quant.Grav. 20, 2417-2428 (2003); D. Birmingham, I. Sachs, S. N. Solodukhin, Phys. Rev. Lett. 88, 151301 (2002); D. Birmingham, Phys.Rev. D 64, 064024 (2001); B. Wang, E. Abdalla and R. B. Mann, Phys. Rev. D 65, 084006 (2002); S. Musiri, G. Siopsis, Phys. Lett. B 576, 309-313 (2003); R. Aros, C. Martinez, R. Troncoso, J. Zanelli, Phys. Rev. D 67, 044014 (2003); A. Nunez, A. O. Starinets, Phys. Rev. D 67, 124013 (2003); B. Wang, C.Y. Lin and C. Molina, Phys. Rev. D 70, 064025 (2004).

[8] E. Abdalla, B. Wang, A. Lima-Santos and W. G. Qiu, Phys. Lett. B 538, 435 (2002); E. Abdalla, K. H. CastelloBranco and A. Lima-Santos, Phys. Rev. D 66, 104018 (2002).

[9] S. Hod, Phys. Rev. Lett. 81, 4293 (1998); A. Corichi, Phys. Rev. D 67, 087502 (2003); L. Motl, Adv. Theor. Math. Phys. 6, 1135-1162 (2003); L. Motl and A. Neitzke, Adv. Theor. Math. Phys. 7, 307-330 (2003); A. Maassen van den Brink, J. Math. Phys. 45, 327 (2004); O. Dreyer, Phys. Rev. Lett. 90, 08130 (2003); G. Kunstatter, Phys. Rev. Lett. 90, 161301 (2003); N. Andersson and C. J. Howls, Class. Quant. Grav. 21, 1623-1642 (2004); V. Cardoso, J. Natario and R. Schiappa, J. Math. Phys. 45 , 4698-4713 (2004); Jose Natario and Ricardo Schiappa, Adv. Theor. Math. Phys. 8, 1001-1131 (2004); V. Cardoso and J. P. S. Lemos, Phys. Rev. D 67, 084020 (2003); K. H. C. Castello-Branco and E. Abdalla, gr-qc/0309090.

[10] G. Koutsoumbas, S. Musiri, E. Papantonopoulos and G. Siopsis, JHEP 0610 (2006) 006; X. P. Rao, B. Wang and G. H. Yang, Phys.Lett.B649, 472 (2007); J.Y. Shen, B. Wang, C.-Y. Lin, R.-G. Cai, R.-K. Su, JHEP 0707,037 (2007).

[11] S. S. Seahra, C. Clarkson, R. Maartens, Phys.Rev.Lett. 94 (2005) 121302; J.Y. Shen, B. Wang, R-K. Su, Phys.Rev. D74 (2006) 044036.

[12] S.A. Hartnoll, Class. Quant. Grav. 26, 224002 (2009).

[13] C.P. Herzog, J. Phys. A 42, 343001 (2009).

[14] G.T. Horowitz, arXiv: 1002.1722 [hep-th].

[15] S.S. Gubser, Phys. Rev. D 78, 065034 (2008).

[16] S.A. Hartnoll, C.P. Herzog, and G.T. Horowitz, Phys. Rev. Lett. 101, 031601 (2008).

[17] R. Gregory, S. Kanno, and J. Soda, J. High Energy Phys. 10, 010 (2009).

[18] Q.Y. Pan, B. Wang, E. Papantonopoulos, J. Oliveria, and A.B. Pavan, Phys. Rev. D 81, 106007 (2010).

[19] X.H. Ge, B. Wang, S.F. Wu, and G.H. Yang, J. High Energy Phys. 08, 108 (2010).

[20] Y.Q. Liu, Q.Y. Pan, B. Wang, and R.G. Cai, Phys. Lett. B 693, 343 (2010).

[21] R.G. Cai, Z.Y. Nie, and H.Q. Zhang, Phys. Rev. D 82, 066007 (2010).

[22] G.T. Horowitz and M.M. Roberts, Phys. Rev. D 78, 126008 (2008).

[23] I. Amado, M. Kaminski, and K. Landsteiner, J. High Energy Phys. 05, 021 (2009).

[24] G. Koutsoumbas, E. Papantonopoulos, and G. Siopsis, J. High Energy Phys. 07, 026 (2009).

[25] K. Maeda, M. Natsuume, and T. Okamura, Phys. Rev. D 79, 126004 (2009).

[26] J. Sonner, Phys. Rev. D 80, 084031 (2009). 
[27] R.G. Cai and H.Q. Zhang, Phys. Rev. D 81, 066003 (2010).

[28] J.L. Jing and S.B. Chen, Phys. Lett. B 686, 68 (2010).

[29] T. Nishioka, S. Ryu, and T. Takayanagi, J. High Energy Phys. 03, 131 (2010).

[30] G. Siopsis and J. Therrien, J. High Energy Phys. 05, 013 (2010).

[31] J.W. Chen, Y.J. Kao, D. Maity, W.Y. Wen, and C.P. Yeh, Phys. Rev. D 81, 106008 (2010).

[32] X. Gao and H.B Zhang, J. High Energy Phys. 08, 075 (2010).

[33] X.M. Kuang, W.J. Li, and Y. Ling, J. High Energy Phys. 12, 069 (2010).

[34] S. Franco, A.M. Garcia-Garcia, and D. Rodriguez-Gomez, J. High Energy Phys. 04, 092 (2010).

[35] S. Franco, A.M. Garcia-Garcia, and D. Rodriguez-Gomez, Phys. Rev. D 81, 041901(R) (2010).

[36] F. Aprile and J.G. Russo, Phys. Rev. D 81, 026009 (2010).

[37] C.P. Herzog, Phys. Rev. D 81, 126009 (2010).

[38] Q.Y. Pan and B. Wang, Phys. Lett. B 693, 159 (2010).

[39] J.L. Jing, L. Wang, Q.Y. Pan, S.B. Chen, Phys.Rev.D83, 066010 (2011); J.L. Jing, S.B. Chen, Phys. Lett. B 686 (2010) 68.

[40] T. K. Dey, Phys. Lett. B 595, 484 (2004); R. G. Cai, D. W. Pang and A. Wang, Phys. Rev. D 70, 124034 (2004).

[41] R.A.Konoplya, Phys.Rev. D66 044009 (2002).

[42] X. He, B. Wang, R.G. Cai, and C.Y. Lin, Phys. Lett. B 688, 230 (2010); R.-G. Cai, X. He, H.-F. Li, H.-Q. Zhang, Phys.Rev.D84,046001 (2011). 